\title{
Abordagens Metodológicas da Experiência dos Usuários Cegos Aplicadas nas Interações Web em Dispositivos Móveis: Uma Revisão Sistemática da Literatura
}

\author{
Tiago Nogueira ${ }^{1}$, Letycia Duarte ${ }^{2}$, Simone Los $^{2}$, Júllian Luz ${ }^{2}$, Deller Ferreira ${ }^{3}$ \\ ${ }^{1}$ Instituto Federal do Tocantins (IFTO) \\ ${ }^{2}$ Instituto Federal de Mato Grosso (IFMT) \\ ${ }^{3}$ Instituto de Informática \\ Universidade Federal de Goiás (UFG) \\ \{tiago.nogueira\}eifto.edu.br
}

\begin{abstract}
With the exponential growth in the scientific interest on assessing the blind users experience in the web environment, it is essential to develop studies that correlate usability features lined up to the accessibility features. At the same time, with technological resources advances and access to information through different devices, there is a gap in the UX investigation in mobile devices, specifically, in the blind users experience. Therefore, this work proposes a systematic literature revision, identifying the main blind users experience methods applied in the mobile devices interaction. Thus, 805 scientific articles were identified through the literature, which approached subjects like usability, blind users experience and mobile devices. Through result extraction, sixteen different applied methods were identified in the blind users experience on mobile devices interaction. It is pointed out the applicability of methods supported by expert reviewers, the observation techniques, trial studies, validation and conformity verification with the Web Content Accessibility Guidelines.
\end{abstract}

Resumo. Com o crescimento exponencial no interesse científico em avaliar a experiência do usuário cego em ambientes na Web, torna-se imperativo trabalhos que correlacionam atributos de usabilidade alinhados às características de acessibilidade. Ao mesmo tempo, com o avanço dos recursos tecnológicos $e$ o acesso às informações por diferentes dispositivos, há uma lacuna nas investigações sobre Experiência do Usuário (UX) em dispositivos móveis, especificamente, na experiência do usuário cego. Assim, este trabalho propõe uma revisão sistemática da literatura, identificando os principais métodos da experiência do usuário cego aplicados nas interações com dispositivos móveis. Dessa forma, foram identificados por meio da literatura, 805 artigos científicos, os quais endereçavam assuntos sobre usabilidade, experiência do usuário cego e dispositivos móveis. Por meio da extração dos resultados, identificou-se dezesseis diferentes métodos aplicados na experiência do usuário cego, nas interações com dispositivos móveis. Destaca-se a aplicabilidade dos métodos apoiados por revisores especialistas, as técnicas de observações, estudos experimentais, validação e verificação de conformidade com as Diretrizes de Acessibilidade do Conteúdo na Web. 


\section{Introdução}

Nos últimos anos, houve um crescimento exponencial no interesse científico em avaliar os aspectos subjetivos na Experiência do Usuário (UX), contribuindo assim para o entendimento nas Interações Humano-Computador (IHC). Ao mesmo tempo, com o avanço dos recursos tecnológicos e o acesso às informações por meio dos dispositivos móveis, há uma lacuna nas investigações sobre UX, especificamente, na experiência do usuário cego em dispositivos móveis [Borg et al. 2015].

Neste sentido, a UX estabelece uma relação com a Usabilidade, por meio de métodos de avaliação de Usabilidade maduros [Law and Abrahão 2014]. No entanto, os métodos de mensuração da UX baseiam-se basicamente, nos métodos consolidados da satisfação dos usuários, da eficiência e eficácia das aplicações e dos mecanismos de Usabilidade [Law and Abrahão 2014].

Segundo [Lallemand et al. 2015], o termo Usabilidade é demasiadamente estreito, não representando uma visão abrangente das interações dos usuários. Assim, a UX abarca além dos conceitos fundamentais da Usabilidade, os aspectos subjetivos das interações, por exemplo, as emoções dos usuários durante as interações em aplicações móveis.

De acordo com [Nogueira et al. 2017], com a mudança conceitual de Usabilidade para a UX, profissionais de IHC encontram novos desafios na evolução do design de interação. Assim, por meio da avaliação dos aspectos subjetivos da UX, em aplicações web, é possível identificar características emocionais que podem colaborar para a construção de interfaces mais acessíveis. Essas emoções podem ser classificadas como UX positiva ou negativa, podendo ser mensuradas em qualquer plataforma, entre elas, em dispositivos móveis.

Para [Tuch et al. 2016], trabalhar com UX negativas torna-se valioso. A compreensão dos determinantes negativos informa aos projetistas sobre as possíveis armadilhas na UX em produtos ou aplicações. Além disso, com uma ampla gama de fenômenos psicológicos, existem evidências de que as experiências negativas têm um impacto mais forte nas pessoas do que as positivas. Em uma estimativa, cinco experiências positivas são necessárias para compensar uma negativa [Tuch et al. 2016].

Não obstante, com o aumento de pessoas com deficiências buscando acesso aos recursos disponibilizados na web, problemas de acessibilidade e de UX de cegos tendem a aumentar [Nogueira 2015].

Assim, este trabalho propõe-se à realização de uma revisão sistemática da literatura, objetivando a identificação dos principais métodos da UX de cegos aplicadas nas interações com dispositivos móveis, publicados nos últimos 5 anos. Para tal, na seção 2 é realizada uma revisão sistemática da literatura; na seção 3 são apresentados os resultados e discussões; e na seção 4 as conclusões.

\section{Revisão Sistemática da Literatura}

Para [Nogueira et al. 2017], nos últimos anos houve um aumento significativo no interesse científico na busca do entendimento da experiência do usuário, principalmente sob os aspectos da acessibilidade na web. Ao mesmo tempo, com o acesso a diferentes tipos de dispositivos por usuários, principalmente usuários com necessidades especiais, surgem 
novos desafios para a comunidade da Interação Humano-Computador (IHC). Neste contexto, surge a seguinte indagação: Q01 - Quais são os principais métodos aplicados na Experiência do Usuário Cegos nas interações com dispositivos móveis?

Dessa forma, para responder à questão de pesquisa Q01, esta revisão sistemática da literatura utilizou-se a abordagem proposta por [Wohlin 2014]. Assim, a subseção 2.1 apresenta a seleção das fontes de pesquisa adotados por esta revisão, a construção do protocolo de pesquisa e os critérios de inclusão e exclusão. Na subseção 2.2 será apresentado a execução desta Revisão Sistemática da Literatura e na subseção 2.3 a extração dos dados.

\subsection{Planejamento da Pesquisa}

Objetivando a identificação de artigos científicos relevantes, os quais endereçam assuntos sobre a experiência dos usuários, usabilidade e dispositivos móveis, as seguintes bases de pesquisa foram adotadas: a) ACM Digital Library; b) Google Scholar; c) IEEE Digital Library; d) Science Direct; e) Springer Link.

Esta revisão sistemática buscou artigos científicos publicados entre janeiro de 2011 a dezembro de 2016, em periódicos e revistas internacionais. Dessa forma, com base na questão de pesquisa (Q01), foram extraídas as seguintes palavras-chaves:"user experience", "usability", "blind user", "mobile". Assim, construiu-se a String de Busca P01 - ("blind" AND “mobile" AND ("user experience" OR "usability”) OR "wcag”).

Com intuito de realizar testes preliminares de eficiência, ao aplicar a String de Busca no Google Scholar, foram identificados 25 artigos, os quais endereçavam assuntos sobre usabilidade e experiência do usuário. Desta forma a String de Busca mostrou-se suficiente para os objetivos desta pesquisa, trazendo-nos informações essenciais a respeito dos métodos mais propícios para a experiência do usuário cego em dispositivos móveis.

O processo de construção dos critérios de inclusão e exclusão deu-se com intuito de selecionar artigos os quais corroboram para resposta da questão de pesquisa Q01, isto é, para rejeição ou aceitação dos estudos. Os critérios de inclusão adotados nesta Revisão Sistemática da literatura foram: a) Trabalhos que utilizam-se de métodos para avaliação da UX de cegos aplicadas nas interações com dispositivos móveis; b) Trabalhos que investigam às experiências de usuários cegos, sob a perspectiva da usabilidade em dispositivos móveis; c) Trabalhos que definem métodos de inclusão a usuários cegos em dispositivos móveis.

Os critérios de exclusão adotados foram: a) Trabalhos que avaliam métodos de usuários que não sejam cegos; b) Trabalhos que apresentam métodos de usuários cegos, mas não em dispositivos móveis; c) Trabalhos que não se encaixam no período requisitado (5 anos); d) Trabalhos que não possuem resumo (abstract); e) Trabalhos que não avaliam métodos de usuários cegos em dispositivos móveis.

\subsection{Execução da Pesquisa}

Por meio da execução do protocolo de pesquisa (P01), foram identificados 805 artigos científicos, os quais endereçavam assuntos sobre usabilidade, experiência do usuário cego e dispositivos móveis. A Figura 1(a) apresenta o quantitativo de artigos científicos identificados por cada base. 


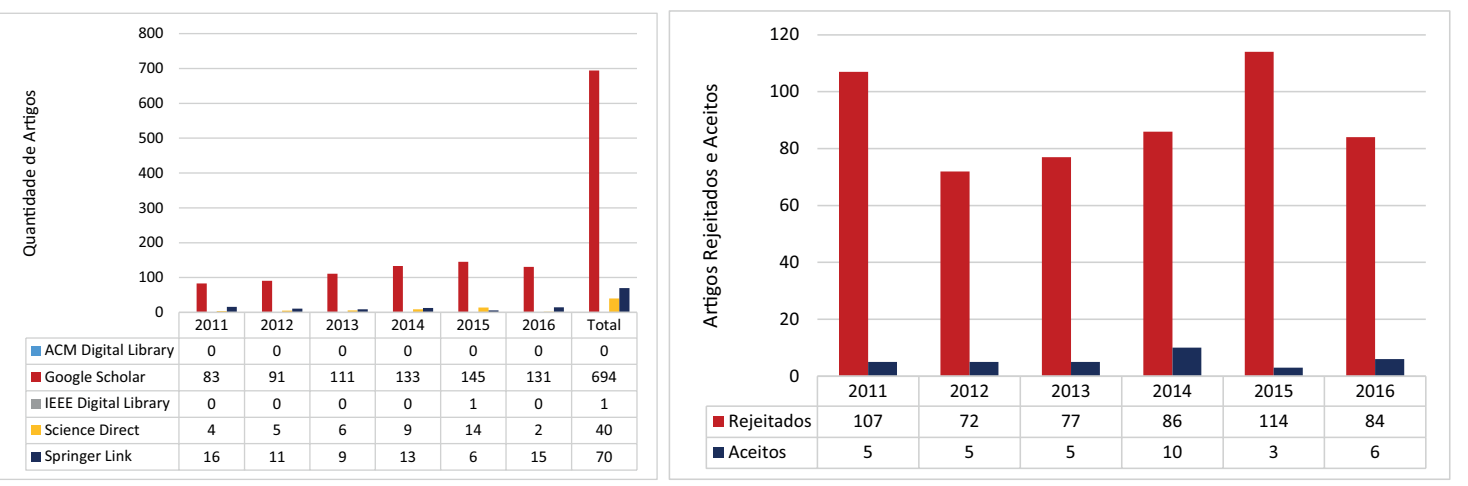

(a) Artigos científicos identificados por (b) Artigos científicos rejeitados/aceitos por período/ano base/fonte

Figura 1. Quantitativo de artigos científicos identificados, rejeitados e aceitos

Foram identificados 694 artigos científicos na base Google Scholar, 1 artigo científico na base IEEE Digital Library, 40 artigos científicos na base Science Direct e 70 artigos científicos na base Springer Link. Observa-se que a quantidade de artigos identificados na base Google Scholar corresponde a $86 \%$ do total de artigos identificados por esta revisão.

Dos 805 artigos científicos identificados, 231 artigos foram classificados como "duplicados" (aproximadamente 28\% da quantidade total dos artigos). Após a exclusão dos artigos duplicados, restaram 574 artigos, dos quais 34 foram "aceitos" e 540 foram "rejeitados". A Figura 1(b) apresenta o quantitativo de artigos que foram rejeitados/aceitos, por meio da aplicação dos critérios de exclusão e inclusão, nesta pesquisa.

Foram rejeitados 107 artigos científicos publicados em 2011, 72 artigos publicados em 2012, 77 artigos publicados em 2013, 86 artigos publicados em 2014, 114 artigos publicados em 2015 e 84 artigos publicados em 2016. Observou-se que, por meio dos critérios de exclusão, cerca de $67 \%$ do total dos artigos identificados foram rejeitados. Este fato ocorreu porque a grande maioria dos artigos científicos não endereçavam assuntos sobre a experiência do usuário cego em dispositivos móveis, apenas assuntos sobre a experiência do usuário vidente (que enxergam). Por meio da aplicação dos critérios de inclusão, foram aceitos 34 artigos. Observa-se que este número representa cerca de 4\% do total de artigos identificados.

Por meio da aplicação dos critérios de inclusão, foram aceitos 5 artigos científicos publicados em 2011, 5 artigos publicados em 2012, 5 artigos publicados em 2013, 10 artigos publicados em 2014, 3 artigos publicados em 2015 e 6 artigos publicados em 2016.

\subsection{Extração dos Dados}

O processo de extração dos dados dos artigos que foram aceitos se deu pela leitura criteriosa por pares de revisores e pela supervisão de um pesquisador/especialista. Dessa forma, foram analisados 34 artigos científicos com intuito de identificar os principais métodos utilizados para a investigação da experiência do usuário cego em dispositivos móveis. A Tabela 1 apresenta os métodos identificados/extraídos por meio das análises/leituras dos artigos. 
Tabela 1. Métodos de UX cegos em dispositivos móveis

\begin{tabular}{|c|c|}
\hline ID & Métodos X Autores \\
\hline 1 & Heurísticas de Usabilidade [Mi et al. 2014]; \\
\hline 2 & \begin{tabular}{lcr} 
Revisão por especialistas & [Pribeanu et al. 2014] & [Neuschmid et al. 2012] \\
[Leporini 2011] & \multicolumn{2}{c}{ [Kerkmann and Lewandowski 2012] } \\
[Bose and Jürgensen 2014] & [Loureiro et al. 2015] & [Mi et al. 2014] \\
[Buzzi et al. 2011] & [Fakrudeen et al. 2014] & [Milne et al. 2014] \\
[Yesilada et al. 2011]; & &
\end{tabular} \\
\hline 3 & $\begin{array}{l}\text { Verificação/Avaliação de conformidade com a WCAG [Pribeanu et al. 2014] } \\
\begin{array}{l}\text { [Neuschmid et al. 2012] [Leporini 2011] [Babu and Singh 2013] } \\
\text { [Murphy and Maycock 2013] [Bose and Jürgensen 2014]; }\end{array}\end{array}$ \\
\hline 4 & $\begin{array}{l}\text { Inspeção por peritos em usabilidade [Leporini 2011] [Pribeanu et al. 2014] } \\
\text { [Neuschmid et al. 2012]; }\end{array}$ \\
\hline 5 & $\begin{array}{l}\text { Estudos experimentais } \quad \text { [Sultan et al. 2015] } \\
\text { [Baumann 2012]; }\end{array}$ \\
\hline 6 & $\begin{array}{lrr}\begin{array}{l}\text { Observação direta } \\
\text { [Baumann 2012] }\end{array} \quad \text { [Kirinic et al. 2016] } & \text { [Ferati et al. 2014] } \\
\text { [Kerkmann and Lewandowski 2012] } & \text { [Francis et al. 2013] } \\
\text { [Schoeberlein and Wang 2012] } & \text { [Bose and Jürgensen 2014] } \\
\text { [Köhlmann and Lucke 2015] } & \text { [Encelle et al. 2011] } \\
\text { [Yesilada et al. 2011] [Ruiz et al. 2011] [Martínez and Pluke 2014]; }\end{array}$ \\
\hline 7 & Verbalização do pensamento/ Verbal Think Aloud [Babu and Singh 2013]; \\
\hline 8 & $\begin{array}{l}\text { Questionários estruturados e abertos [Babu and Singh 2013] } \\
\text { [Francis et al. 2013] [Schoeberlein and Wang 2012]; }\end{array}$ \\
\hline 9 & $\begin{array}{l}\text { Análise de dados de entrada (microfone/teclado) } \\
\text { [Façanha et al. 2014] [Ismirle et al. 2016]; }\end{array}$ \\
\hline 10 & $\begin{array}{l}\text { Frameworks de Usabilidade/Acessibilidade [Angkananon et al. 2014] } \\
\text { [Francis et al. 2013]; }\end{array}$ \\
\hline 11 & \begin{tabular}{lcc} 
Testes com Usuários & [Sierra et al. 2012] & [Francis et al. 2013] \\
[Bose and Jürgensen 2014] & \multicolumn{2}{c}{ [Schoeberlein and Wang 2012] } \\
[Köhlmann and Lucke 2015] & [Ismirle et al. 2016] & [Loureiro et al. 2015] \\
[Ismirle et al. 2016] & [Façanha et al. 2014] & [Pascual et al. 2014] \\
[Sahasrabudhe and Singh 2016] [Yesilada et al. 2011]; &
\end{tabular} \\
\hline 12 & $\begin{array}{l}\text { Modelos/Modelagem de Comportamentos/ } \\
\text { [Murphy and Maycock 2013] [Loiacono et al. 2013]; }\end{array}$ \\
\hline 13 & 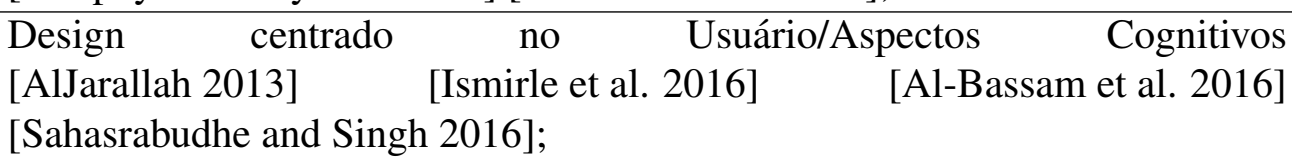 \\
\hline 14 & $\begin{array}{l}\text { Prototipagem [Köhlmann and Lucke 2015] [Ismirle et al. 2016] } \\
\text { [Al-Bassam et al. 2016] [Ismirle et al. 2016] [Fakrudeen et al. 2014]; }\end{array}$ \\
\hline 15 & $\begin{array}{l}\text { Estudo empírico/ } \\
\text { [Pribeanu et al. 2014]; }\end{array}$ \\
\hline 16 & Método participativo [Mi et al. 2014]. \\
\hline
\end{tabular}


Por meio da extração dos dados, foram identificados 16 diferentes métodos aplicados nos processos, de forma metodológica, nas experiências de usuários cegos em dispositivos móveis: revisão por especialistas; verificação/avaliação de conformidade com a WCAG; estudo empírico/social empírico; inspeção por peritos em usabilidade; estudos experimentais; observação direta; verbalização do pensamento/ verbal think aloud; questionários estruturados e abertos; análise de dados de entrada (microfone/teclado); frameworks de usabilidade/acessibilidade; testes com usuários; modelos/modelagem de comportamentos/ acessibilidade; design centrado no usuário/aspectos cognitivos; prototipagem; heurísticas de usabilidade; método participativo.

\section{Resultados e Discussões}

Nesta seção foram avaliados os métodos encontrados na Extração de Dados (subseção 2.3). Dentre os 16 métodos descobertos, destacou-se o método revisão por especialistas [Pribeanu et al. 2014] [Neuschmid et al. 2012] [Leporini 2011] [Kerkmann and Lewandowski 2012] [Bose and Jürgensen 2014] [Loureiro et al. 2015] [Mi et al. 2014] [Buzzi et al. 2011] [Fakrudeen et al. 2014] [Milne et al. 2014] [Yesilada et al. 2011]. Este método tem como objetivo identificar problemas de usabilidade para a pessoa com deficiência visual, visando tanto a acessibilidade quanto a usabilidade. O método verificação de conformidade de acordo com as diretrizes de acessibilidade (WCAG), possui o objetivo de disponibilizar uma visão geral das violações de acessibilidade e fornecer dicas úteis para revisores especialistas [Pribeanu et al. 2014] [Neuschmid et al. 2012] [Leporini 2011] [Babu and Singh 2013] [Murphy and Maycock 2013] [Bose and Jürgensen 2014].

O método social empírico, em estreita colaboração com o grupo-alvo e especialistas, conta com a aplicação de questionários estruturados [Neuschmid et al. 2012]. Os questionários são compostos por 5 seções com 55 perguntas. Neste método, é realizado um estudo empírico sobre os níveis de acessibilidade e a experiência do usuário cego, analisado do ponto de vista dos especialistas [Neuschmid et al. 2012] [Pribeanu et al. 2014].

Outro método identificado, por meio da extração dos dados dos artigos, inspeção por peritos em usabilidade, trata-se da avaliação da usabilidade por meio da aplicação dos princípios de estruturação do conteúdo, adequação do conteúdo e interatividade [Leporini 2011]. O princípio de estruturação do conteúdo está relacionado à partição lógica dos elementos de interface, por exemplo, o número de links da interface. Os princípios de adequação do conteúdo estão relacionados aos números de links apropriados, nomes apropriados para tabelas e imagens. A interatividade está relacionada à operabilidade da interface via teclados, sobre os mecanismos de mensagens e gestão dos dados [Leporini 2011].

Para [Sultan et al. 2015], por meio de estudos experimentais, pode-se avaliar aspectos da usabilidade em interfaces de dispositivos móveis por usuários cegos. Desta forma, avaliando a usabilidade de três telefones móveis, com aplicações Android distintas, é possível mensurar o grau de facilidade de uso, de aprendizado, da eficiência e compreensão dos usuários cegos durante as interações em diferentes aplicações [Sultan et al. 2015].

O método de observação direta tem como finalidade a realização da coleta de relatórios verbais de estudantes cegos 
[Babu and Singh 2013] [Ferati et al. 2014] [Baumann 2012] [Kirinic et al. 2016] [Francis et al. 2013] [Kerkmann and Lewandowski 2012] [Bose and Jürgensen 2014] [Schoeberlein and Wang 2012] [Encelle et al. 2011] [Köhlmann and Lucke 2015] [Loureiro et al. 2015] [Milne et al. 2014] [Yesilada et al. 2011] [Ruiz et al. 2011] [Martínez and Pluke 2014]. Assim, os participantes trabalham em uma única tarefa e simultaneamente são verbalizados os pensamentos dos participantes durante as interações [Babu and Singh 2013].

Aplicando-se da observação direta, pode-se, por meio do método de avaliação da WCAG, usando apenas a análise de texto, interpretar e explicar os principais comportamentos dos usuários cegos nas interações em aplicações móveis. Assim, para obter melhores resultados, aplicam-se questionários estruturados e abertos [Babu and Singh 2013] [Francis et al. 2013] [Schoeberlein and Wang 2012].

Por meio da análise de dados de entrada do teclado, é possível identificar e avaliar a experiência do usuário nos processos de testes de usabilidade. Assim, pode-se utilizar dos componentes de entrada, teclado e microfone, para realizar observações do comportamento dos usuários durante as interações [Miao et al. 2016].

Observou-se, por meio das análises, a identificação de métodos que auxiliam aos desenvolvedores na criação de interfaces acessíveis, melhorando assim, a usabilidade das aplicações por usuários cegos [Angkananon et al. 2014]. Neste sentido, pode-se utilizar de frameworks para compreender os problemas e soluções enfrentadas por pessoas cegas [Angkananon et al. 2014] [Francis et al. 2013]. Assim, o Framework de Interatividade Aprimorada das Tecnologias (TEIF), consiste em 19 perguntas de múltipla escolha, o qual orienta ao desenvolvedor na construção de uma interface acessível e universal [Angkananon et al. 2014].

\section{Conclusões}

Esta revisão sistemática da literatura incidiu sobre as publicações científicas entre 2011 a 2016. Desta forma, foram identificados 805 artigos que endereçavam métodos da experiência do usuário cego aplicados nas interações com dispositivos móveis. Aplicando os critérios de inclusão e exclusão adotados nesta revisão, foram selecionados 34 artigos.

Percebeu-se, por meio da extração dos dados dos artigos, a identificação de 16 diferentes métodos aplicados na experiência do usuário cego, nas interações com dispositivos móveis. Assim, destacam-se a aplicabilidade dos métodos apoiados por revisores especialistas, empregando as técnicas de observações diretas, por meio de estudos experimentais, verificando e avaliando a conformidade com a WCAG. Observou-se também, as implementações de frameworks, objetivando a construção de interfaces acessíveis e com alto índice de usabilidade.

Não obstante, há um consenso entre os trabalhos, em processos de usabilidade e acessibilidade, ao mensurar a experiência do usuário cego, em utilizar-se da aplicabilidade de tarefas e questionários, por meio dos testes com usuários, avaliando o comportamento destes durante as interações. Neste sentido, percebeu-se o uso dos métodos de modelos comportamentais, os quais realizam a modelagem das interações dos usuários em determinadas aplicações.

Portanto, por meio da identificação dos principais métodos aplicados nos proces- 
sos de mensuração da experiência do usuário cego em dispositivos móveis, os pesquisadores da área da Interação Humano-Computador (IHC) podem selecionar os métodos mais adequados, de acordo com seus objetivos, utilizando-se das melhores práticas da usabilidade e da acessibilidade na web.

\section{Referências}

Al-Bassam, D., Alotaibi, H., Alotaibi, S., and Al-Khalifa, H. S. (2016). Easytrans: Accessible translation system for blind translators. In International Conference on Computers Helping People with Special Needs, pages 583-586. Springer.

AlJarallah, K. (2013). Cognitive user-centred design approach to improve accessibility for blind people during online interaction.

Angkananon, K., Wald, M., and Gilbert, L. (2014). Applying technology enhanced interaction framework to accessible mobile learning. Procedia Computer Science, 27:261270.

Babu, R. and Singh, R. (2013). Enhancing learning management systems utility for blind students: A task-oriented, user-centered, multi-method evaluation technique. Journal of Information Technology and Education: Research, 12.

Baumann, K. (2012). Barrierefreiheit von facebook: Untersuchung mit hilfe des bitvtests.

Borg, J., Lantz, A., and Gulliksen, J. (2015). Accessibility to electronic communication for people with cognitive disabilities: a systematic search and review of empirical evidence. Universal Access in the Information Society, 14(4):547-562.

Bose, R. and Jürgensen, H. (2014). Accessibility of e-commerce websites for visionimpaired persons. In International Conference on Computers for Handicapped Persons, pages 121-128. Springer.

Buzzi, M. C., Buzzi, M., Leporini, B., and Martusciello, L. (2011). Making visual maps accessible to the blind. In International Conference on Universal Access in HumanComputer Interaction, pages 271-280. Springer.

Encelle, B., Ollagnier-Beldame, M., Pouchot, S., and Prié, Y. (2011). Annotation-based video enrichment for blind people: A pilot study on the use of earcons and speech synthesis. In The proceedings of the 13th international ACM SIGACCESS conference on Computers and accessibility, pages 123-130. ACM.

Façanha, A. R., Viana, W., Pequeno, M. C., de Borba Campos, M., and Sánchez, J. (2014). Touchscreen mobile phones virtual keyboarding for people with visual disabilities. In International Conference on Human-Computer Interaction, pages 134-145. Springer.

Fakrudeen, M., Yousef, S., and Hussein, A. H. (2014). Analyzing app inventor for building usable touch screen courseware for blind users.

Ferati, M., Raufi, B., Kurti, A., and Vogel, B. (2014). Accessibility requirements for blind and visually impaired in a regional context: An exploratory study. In Usability and Accessibility Focused Requirements Engineering (UsARE), 2014 IEEE 2nd International Workshop on, pages 13-16. IEEE. 
Francis, H., Al-Jumeily, D., and Lund, T. O. (2013). A framework to support e-commerce development for people with visual impairment. In Developments in eSystems Engineering (DeSE), 2013 Sixth International Conference on, pages 335-341. IEEE.

Ismirle, J., O Bara, I., Swierenga, S. J., and Jackson, J. E. (2016). Touchscreen voting interface design for persons with disabilities insights from usability evaluation of mobile voting prototype. In Proceedings of the Human Factors and Ergonomics Society Annual Meeting, volume 60, pages 780-784. SAGE Publications.

Kerkmann, F. and Lewandowski, D. (2012). Accessibility of web search engines: Towards a deeper understanding of barriers for people with disabilities. Library Review, 61(8/9):608-621.

Kirinic, V., Kozina, M., and Vidacek-Hains, V. (2016). Accessibility of information: International standards, recomendations and practices. Economic and Social Development: Book of Proceedings, page 47.

Köhlmann, W. and Lucke, U. (2015). Alternative concepts for accessible virtual classrooms for blind users. In Advanced Learning Technologies (ICALT), 2015 IEEE 15th International Conference on, pages 413-417. IEEE.

Lallemand, C., Gronier, G., and Koenig, V. (2015). User experience: A concept without consensus? exploring practitioners' perspectives through an international survey. Computers in Human Behavior, 43:35-48.

Law, E. L.-C. and Abrahão, S. (2014). Interplay between user experience (ux) evaluation and system development.

Leporini, B. (2011). Google news: how user-friendly is it for the blind? In Proceedings of the 29th ACM international conference on Design of communication, pages 241-248. ACM.

Loiacono, E. T., Djamasbi, S., and Kiryazov, T. (2013). Factors that affect visually impaired users' acceptance of audio and music websites. International Journal of HumanComputer Studies, 71(3):321-334.

Loureiro, J. R., Cagnin, M. I., and Paiva, D. M. B. (2015). Analysis of web accessibility in social networking services through blind users' perspective and an accessible prototype. In International Conference on Computational Science and Its Applications, pages 117-131. Springer.

Martínez, L. and Pluke, M. (2014). Mandate m 376: new software accessibility requirements. Procedia Computer Science, 27:271-280.

Mi, N., Cavuoto, L. A., Benson, K., Smith-Jackson, T., and Nussbaum, M. A. (2014). A heuristic checklist for an accessible smartphone interface design. Universal access in the information society, 13(4):351-365.

Miao, M., Pham, H. A., Friebe, J., and Weber, G. (2016). Contrasting usability evaluation methods with blind users. Universal Access in the Information Society, 15(1):63-76.

Milne, L. R., Bennett, C. L., and Ladner, R. E. (2014). The accessibility of mobile health sensors for blind users.

Murphy, M. P. and Maycock, K. (2013). Evaluating web accessibility for blind individuals. 
Neuschmid, J., Hennig, S., Schrenk, M., Wasserburger, W., and Zobl, R. (2012). Barrierefreiheit von online Stadtplänen-das Beispiel AccessibleMap. na.

Nogueira, T. C., Ferreira, D. J., Carvalho, S. T., and Berreta, L. O. (2017). Evaluating responsive web design's impact on blind users. IEEE MultiMedia, 24(2):86-95.

Nogueira, T. d. C. (2015). Estudo comparativo da experiência de usuários cegos e videntes no design web responsivo e não responsivo.

Pascual, A., Ribera, M., Granollers, T., and Coiduras, J. L. (2014). Impact of accessibility barriers on the mood of blind, low-vision and sighted users. Procedia Computer Science, 27:431-440.

Pribeanu, C., Fogarassy-Neszly, P., and Pătru, A. (2014). Municipal web sites accessibility and usability for blind users: preliminary results from a pilot study. Universal access in the information society, 13(3):339-349.

Ruiz, B., Pajares, J. L., Utray, F., and Moreno, L. (2011). Design for all in multimedia guides for museums. Computers in Human Behavior, 27(4):1408-1415.

Sahasrabudhe, S. and Singh, R. (2016). Accessibility problems of blind mhealth users, a pilot study.

Schoeberlein, J. G. and Wang, Y. (2012). Accessible collaborative writing for persons who are blind: a usability study. In Proceedings of the 14th international ACM SIGACCESS conference on Computers and accessibility, pages 267-268. ACM.

Sierra, J., De Togores, J. S. R., and Selva, J. (2012). Designing mobile apps for visually impaired and blind users. In The Fifth International Conference on Advances in Computer-Human Interactions, pages 47-52. Citeseer.

Sultan, N., Siddiq, K., Rashid, T., and Farooque, M. (2015). Evaluation of smart phone applications accessibility for blind users. Evaluation, 127(3).

Tuch, A. N., Schaik, P. V., and Hornbæk, K. (2016). Leisure and work, good and bad: The role of activity domain and valence in modeling user experience. ACM Transactions on Computer-Human Interaction (TOCHI), 23(6):35.

Wohlin, C. (2014). Guidelines for snowballing in systematic literature studies and a replication in software engineering. In Proceedings of the 18th international conference on evaluation and assessment in software engineering, page 38. ACM.

Yesilada, Y., Brajnik, G., and Harper, S. (2011). Barriers common to mobile and disabled web users. Interacting with Computers, 23(5):525-542. 